Proceedings of the ASME 2015 34th International Conference on Ocean, Offshore and Arctic Engineering

May 31-June 5, 2015, St. John's, Newfoundland, Canada

OMAE2015-41971

\title{
PROGRESSES IN THE DEVELOPMENT OF A WEAKLY-NONLINEAR WAVE BODY INTERACTION MODEL BASED ON THE WEAK-SCATTERER APPROXIMATION
}

\author{
Camille Chauvignéa,b \\ Guillaume Ducrozeta
}

\author{
Lucas Letournela \\ Pauline Bozonnet ${ }^{\mathrm{b}}$ \\ Pierre Ferrant ${ }^{a}$ \\ a Ecole Centrale de Nantes, LHEEA \\ UMR CNRS 6598 - Nantes, France \\ b IFP Energies Nouvelles, \\ Rueil-Malmaison, France
}

\author{
Aurélien Babarit ${ }^{a}$ \\ Jean-Christophe Gilloteaux ${ }^{a}$
}

\section{ABSTRACT}

In continuation of [1], this paper presents the progress made towards the development of a new modeling tool based on the Weak-Scatterer approaches . Recent developments are the coupling of the fluid and body solver in order to predict the free motion response of the body. Pressure field over the wetted area is obtained by solving an additional boundary value problem for the time derivative of the velocity potential. Tanizawa's [2] and Cointe's [3] formulations for the acceleration condition on the body are revisited. Numerical prediction with the present method for a submerged body in vertical free motion is presented and energy conservation is verified. In order to adapt the mesh to the moving body geometry, advanced mesh moving schemes have been integrated based on radial basis functions [4] and spring analogy methods. In this way it is possible to solve the problem with an Arbitrary Euler Lagrangian formalism and preserve the order of the numerical scheme. However moving mesh methods are limited in time and automatic remeshing generation algorithms have been integrated in order to enable simulating longer durations. Finally, comparisons of wave diffraction and radiation predicted by linear theory, a fully nonlinear BEM solver and the present method are shown.

\section{INTRODUCTION}

Recent developments in marine renewable energy are facing some new challenges in the field of fluid-structure interactions and seakeeping analysis. Linear models are not well suited for wave energy converter system because they are limited to small amplitude. On the contrary, wave energy converters are submitted to large amplitude motion because of their size and conception. In consequence results obtained with linear model may fail in the prediction of the body motion response and the estimation of the quantity of energy that can be extracted. CFD models are able to model the body response in large amplitude motion solving RANS equations but involve large CPU time requirement (typically hours of CPU time per wave period [5]) and are thus limited to the study of wave energy converter design. Several fully nonlinear potential solvers have been developed in the last years [6]. No linear approximation is made in such models and large amplitude motion can be simulated with the exact free surface position and exact position of the floating system. The boundaries of the fluid domain are also unknowns in these models. Its accurate estimation is more efficient than CFD solvers but remains time consuming and present some weaknesses in terms of robutness. 
A weakly-nonlinear approach based on the Weak-Scatterer equation [7] may be a good alternative. In such approach, the flow velocity potential is decomposed in the incident potential and the scattered potential (radiated and diffracted waves). The scattered potential is assumed to be small. Consequently, free surface conditions can be linearized around the incident wave elevation. No restrictions are made on the incident wave potential (non linear steep wave may be considered) and the body motion amplitude (the body may undergo large amplitude of oscillations). This approach seems more robust than the fully nonlinear one because the position of the free surface is known at each time step (Taylor expansion of the free surface conditions is done around incident free surface elevation).

In this work, an Arbitrary Lagrangian-Eulerian method (ALE) is used together with efficient algorithms for dealing with the mesh deformations. For free body motion response, the time derivative of the velocity potential (instantaneous pressure) over the wetted surface is an additional unknown to the problem that is solved by application of Green's second identity equation. Both Tanizawa's [2] and Cointe's [3] formulations for the body condition are used in this case.

In the first part of this paper, mathematical, numerical aspects and simulation results for the free motion of a submerged body in waves are presented. In the second part, efficient algorithms for mesh management in case of surface piercing bodies are presented and discussed. Then, the model is validated against litterature results for the diffraction of a fixed free surface piercing vertical cylinder. For large steepness, nonlinear effects become important [8] and linear models generally underestimate the runup around the structure. Comparisons of runups around the cylinder are compared to linear model, nonlinear model and experimental data. Finally, results for the radiation of a surging and pitching surface piercing vertical cylinder are shown.

\section{THEORY}

\section{Potential Theory and Weak-Scatterer Approximation}

Assuming incompressible and inviscid fluid with irrotational flow, the flow velocity derives from a velocity potential $\phi$ which satisfies the Laplace equation.

$$
\nabla^{2} \phi(x, y, z, t)=0
$$

in the fluid domain, $D$, with a boundary, $\Gamma$. Using Green's second identity, the velocity potential can be written as the solution of the boundary integral equation (BIE).

$\alpha\left(\mathbf{x}_{l}\right) \phi\left(\mathbf{x}_{l}\right)=\iint_{\Gamma}\left[\frac{\partial \phi}{\partial n}(\mathbf{x}) G\left(\mathbf{x}, \mathbf{x}_{l}\right)-\phi(\mathbf{x}) \frac{\partial G}{\partial n}\left(\mathbf{x}, \mathbf{x}_{l}\right)\right] d \Gamma \quad \forall \mathbf{x}_{l} \in \Gamma$ where $G$ is the Rankine singularity, $\alpha$ is the interior solid angle and $\mathbf{x}$ and $\mathbf{x}_{l}$ are vectors positions.

The Weak-Scatterer (WS) approximation [1] is used to solve the fluid-structure interaction problem. The flow solution $(\phi, \eta)$ is split into an incident and scattered components. The scattered component (perturbation) is supposed small compared to the incident component.

$$
\left\{\begin{array}{l}
\phi=\phi_{0}+\phi_{p}, \phi_{p} \ll \phi_{0} \\
\eta=\eta_{0}+\eta_{p}, \eta_{p} \ll \eta_{0}
\end{array}\right.
$$

\section{Boundary Conditions}

The dynamic and kinematic free-surface conditions are

$$
\begin{cases}\frac{\partial \phi}{\partial t}=-\frac{1}{2} \nabla \phi \cdot \nabla \phi-g \eta, & \text { for } z=\eta \\ \frac{\partial \eta}{\partial t}=\frac{\partial \phi}{\partial z}-\nabla \phi \cdot \nabla \eta & , \text { for } z=\eta\end{cases}
$$

A particular time derivative is defined to follow the nodes on the incident free surface elevation

$$
\frac{D_{0}}{D t}=\frac{\partial}{\partial t}+\mathbf{v}_{m e s h} \cdot \nabla
$$

The velocity $\mathbf{v}_{\text {mesh }}$ is defined such as the free surface nodes follow the body in its horizontal motions and such as they follow vertically the motion of the incident wave elevation. Note that for a fully submerged body, the horizontal components of $\mathbf{v}_{\text {mesh }}$ are set to 0 for convenience, leading to : $\mathbf{v}_{\text {mesh }}=\frac{\partial \eta_{0}}{\partial t} \mathbf{z}$. Combining the WS approximation with this particular derivative leads to the following equations for the free-surface conditions :

$$
\begin{aligned}
\frac{D_{0} \eta_{p}}{D t}= & -\frac{\partial \eta_{0}}{\partial t}+\frac{\partial \phi_{p}}{\partial z}+\frac{\partial \phi_{0}}{\partial z}-\nabla \phi_{0} \cdot \nabla \eta_{0} \\
& -\nabla \phi_{p} \cdot \nabla \eta_{0}-\left(\nabla \phi_{0}-\mathbf{v}_{m e s h}\right) \cdot \nabla \eta_{p} \\
& +\eta_{p}\left(\frac{\partial^{2} \phi_{0}}{\partial^{2} z}-\frac{\partial}{\partial z} \nabla \phi_{0} \cdot \nabla \eta_{0}\right)-v \eta_{p} \\
\frac{D_{0} \phi_{p}}{D t}= & -\frac{\partial \phi_{0}}{\partial t}-g\left(\eta_{p}+\eta_{0}\right) \\
& -\frac{1}{2} \nabla \phi_{0}^{2}-\left(\nabla \phi_{0}-v_{m e s h}\right) \cdot \nabla \phi_{p} \\
& -\eta_{p}\left(\frac{\partial^{2} \phi_{0}}{\partial z \partial t}+\frac{\partial}{\partial z} \nabla \phi_{0} \cdot \nabla \phi_{0}\right)-v \phi_{p}
\end{aligned}
$$

where $v$ is a damping coefficient to model the absorbing beach 
The body condition is based on the impermeability of the body surface, where $\mathbf{x}_{\mathbf{B}}$ is the center of mass of the body and $\mathbf{n}$ is the normal on the control point

$$
\frac{\partial \phi_{p}}{\partial n}=-\frac{\partial \phi_{0}}{\partial n}+\left(\frac{D \mathbf{x}_{\mathbf{B}}}{D t}+\omega \times\left(\mathbf{x}-\mathbf{x}_{\mathbf{B}}\right)\right) \cdot \mathbf{n}
$$

At infinity, a radiation condition needs to be satisfied

$$
\left\{\begin{array}{l}
\eta_{p} \rightarrow 0 \\
\phi_{p} \rightarrow 0
\end{array}, \text { when } r \rightarrow \infty\right.
$$

\section{Hydrodynamic Force and Body Motions}

The hydrodynamic pressure on the body is given using Bernoulli's law

$$
p=-\rho\left(\frac{\partial \phi}{\partial t}+\frac{1}{2} \nabla \phi \cdot \nabla \phi+g z\right)
$$

where $\rho$ is the fluid density and $g$ is the gravitational acceleration.

The hydrodynamic force is

$$
\left\{\begin{array}{l}
\mathbf{F}_{H}=\iint_{S_{B}} p \mathbf{n} d S \\
\mathbf{M}_{H}=\iint_{S_{B}} p(\mathbf{r} \times \mathbf{n}) d S
\end{array}\right.
$$

with $S_{B}$ the body immerged surface delimited by the incident wave surface elevation and $\mathbf{r}=\mathbf{x}-\mathbf{x}_{\mathbf{B}}$.

The body motions are obtained according to Newton's law :

$$
\left\{\begin{array}{l}
M \cdot \ddot{\mathbf{x}}_{B}=\mathbf{F}_{H}+\sum \mathbf{F}_{e x t} \\
I \cdot \ddot{\theta}_{B}=\mathbf{M}_{H}+\sum \mathbf{M}_{e x t}
\end{array}\right.
$$

with $\mathbf{F}_{e x t}$ and $\mathbf{M}_{e x t}$ being the external force and moment respectively.

\section{PART I : SUBMERGED BODY IN FREE MOTION Acceleration Potential and Implicit Condition Methods}

The calculation of the hydrodynamic pressure on the body requires the potential time derivative. To obtain this quantity, a finite difference scheme can be used if the body motions are forced, however this method is not precise enough for a freely moving body. Thus a method based on the resolution of a second
BVP for $\frac{\partial \phi}{\partial t}$, called Acceleration Potential Method, is used. The Laplace equation is satisfied by the velocity potential, thus by its partial time derivative as well. The boundary conditions are also of the same kind : Dirichlet for the Free-Surface and Neumann for the body.

$$
\begin{cases}\triangle \frac{\partial \phi}{\partial t}=0 & \text { in the fluid domain } \\ \frac{\partial \phi}{\partial t}=\frac{D_{0 z} \phi}{D t}-\frac{\partial \phi_{0}}{\partial z} \frac{\partial \phi}{\partial z} & \text { on the Free-Surface } \\ \frac{\partial^{2} \phi}{\partial \eta \partial t}=\ddot{\mathbf{x}}_{B} \cdot \mathbf{n}+\left(\ddot{\theta}_{B} \times \mathbf{r}\right) \cdot \mathbf{n}+q & \text { on a body in free motions } \\ \frac{\partial^{2} \phi}{\partial n \partial t}=0 & \text { on fixed boundaries }\end{cases}
$$

The body condition had several expressions depending on the acceleration used. First, Cointe [3] gave a 2D expression based on the acceleration of the body particle, $\ddot{\mathbf{x}}$

$$
\begin{aligned}
\frac{\partial^{2} \phi}{\partial n \partial t} & =\ddot{\mathbf{x}} \cdot \mathbf{n}+\dot{\theta}\left(\dot{\mathbf{x}} \cdot \mathbf{s}-\frac{\partial \phi}{\partial s}\right)-\left(\frac{1}{R} \frac{\partial \phi}{\partial s}+\frac{\partial^{2} \phi}{\partial n \partial s}\right) \dot{\mathbf{x}} \cdot \mathbf{s} \\
& +\left(\frac{\partial^{2} \phi}{\partial s^{2}}-\frac{1}{R} \frac{\partial \phi}{\partial n}\right) \dot{\mathbf{x}} \cdot \mathbf{n}
\end{aligned}
$$

with $\mathbf{s}$ the local tangent vector and $R$ the curvature radius associated to this vector. Then Van Dalen [9] expressed it in 3D, using the same method

$$
\begin{aligned}
\frac{\partial^{2} \phi}{\partial n \partial t}= & \ddot{\mathbf{x}} \cdot \mathbf{n}+\left(\dot{\mathbf{x}} \cdot \mathbf{s}_{1}-\frac{\partial \phi}{\partial s_{1}}\right) \cdot \dot{\theta} \cdot \mathbf{s}_{2}-\left(\dot{\mathbf{x}} \cdot \mathbf{s}_{2}-\frac{\partial \phi}{\partial s_{2}}\right) \cdot \dot{\theta} \cdot \mathbf{s}_{1} \\
& -\left(\frac{1}{R_{1}} \cdot \frac{\partial \phi}{\partial s_{1}}-\frac{\partial^{2} \phi}{\partial s_{1} \partial n}\right) \cdot \dot{\mathbf{x}} \cdot \mathbf{s}_{1}-\left(\frac{1}{R_{2}} \cdot \frac{\partial \phi}{\partial s_{2}}-\frac{\partial^{2} \phi}{\partial s_{2} \partial n}\right) \cdot \dot{\mathbf{x}} \cdot \mathbf{s}_{2} \\
& +\left(\frac{\partial^{2} \phi}{\partial s_{1}^{2}}+\frac{\partial^{2} \phi}{\partial s_{2}^{2}}-\left(\frac{1}{R_{1}}+\frac{1}{R_{2}}\right) \cdot \frac{\partial \phi}{\partial n}\right) \cdot \dot{\mathbf{x}} \cdot \mathbf{n}
\end{aligned}
$$

with also $\left(\mathbf{s}_{1}, \mathbf{s}_{2}\right)$ the local tangent vectors and $\left(R_{1}, R_{2}\right)$ the curvature radius associated to these vectors.

In the meantime, Tanizawa [2] developped a 2D expression based on the acceleration of a fluid particle sliding on the body, $\mathbf{a}$

$$
\frac{\partial^{2} \phi}{\partial n \partial t}=\mathbf{a} \cdot \mathbf{n}+\frac{1}{R}\left(\frac{\partial \phi^{2}}{\partial s}+\frac{\partial \phi^{2}}{\partial n}\right)-\frac{\partial \phi}{\partial s} \frac{\partial^{2} \phi}{\partial s \partial n}+\frac{\partial \phi}{\partial n} \frac{\partial^{2} \phi}{\partial s^{2}}
$$

which was extended in 3D by Berkvens [10]

$$
\begin{aligned}
\frac{\partial^{2} \phi}{\partial n \partial t} & =\mathbf{a} \cdot \mathbf{n}+\left(\frac{1}{R_{1}}+\frac{1}{R_{2}}\right) \frac{\partial \phi^{2}}{\partial n}+\frac{1}{R_{1}} \frac{\partial \phi^{2}}{\partial s_{1}}-\frac{1}{R_{2}} \frac{\partial \phi^{2}}{\partial s_{2}} \\
& +\left(\frac{\partial^{2} \phi}{\partial s_{1}^{2}}+\frac{\partial^{2} \phi}{\partial s_{2}^{2}}\right) \frac{\partial \phi}{\partial n}-\frac{\partial \phi}{\partial s_{1}} \frac{\partial^{2} \phi}{\partial s_{1} \partial n}-\frac{\partial \phi}{\partial s_{2}} \frac{\partial^{2} \phi}{\partial s_{2} \partial n}(17)
\end{aligned}
$$


Developments of all theses expressions were ascertained in this study and their equivalence proved, taking care particularly of the nature of the local base, direct or indirect, used in the different developments. The equivalence also gave additional expressions

$$
\left\{\begin{array}{l}
\frac{\partial^{2} \phi}{\partial s_{1} \partial n}=-\left(\dot{\theta} \cdot \mathbf{s}_{2}\right)-\frac{1}{R_{1}}\left(\dot{\mathbf{x}} \cdot \mathbf{s}_{1}\right) \\
\frac{\partial^{2} \phi}{\partial s_{2} \partial n}=\left(\dot{\theta} \cdot \mathbf{s}_{1}\right)-\frac{1}{R_{2}}\left(\dot{\mathbf{x}} \cdot \mathbf{s}_{2}\right)
\end{array}\right.
$$

Thus a new expression is given here, based on the acceleration of the center of gravity of the body, to be used directly in the Implicit Condition method.

$$
\begin{aligned}
\frac{\partial^{2} \phi}{\partial n \partial t}= & \ddot{\mathbf{x}} \cdot \mathbf{n}+\left(\dot{\theta} \cdot \mathbf{s}_{2}\right)\left(2\left(\dot{\mathbf{x}} \cdot \mathbf{s}_{1}\right)-\frac{\partial \phi}{\partial s_{1}}\right)-\left(\dot{\theta} \cdot \mathbf{s}_{1}\right)\left(2\left(\dot{\mathbf{x}} \cdot \mathbf{s}_{2}\right)-\frac{\partial \phi}{\partial s_{2}}\right) \\
& +\frac{\left(\dot{\mathbf{x}} \cdot \mathbf{s}_{1}\right)}{R_{1}}\left(\frac{\partial \phi}{\partial s_{1}}-\left(\dot{\mathbf{x}} \cdot \mathbf{s}_{1}\right)\right)+\frac{\left(\dot{\mathbf{x}} \cdot \mathbf{s}_{2}\right)}{R_{2}}\left(\frac{\partial \phi}{\partial s_{2}}-\left(\dot{\mathbf{x}} \cdot \mathbf{s}_{2}\right)\right) \\
& +(\dot{\mathbf{x}} \cdot \mathbf{n})\left(\frac{\partial^{2} \phi}{\partial s_{1}^{2}}+\frac{\partial^{2} \phi}{\partial s_{2}^{2}}+\left(\frac{1}{R_{1}}+\frac{1}{R_{2}}\right) \frac{\partial \phi}{\partial n}\right)
\end{aligned}
$$

One can see that this body condition requires the body acceleration, also unknown for a freely moving body. The Implicit Condition method, introduced by Van Dalen [9] and Tanizawa [2], is thus solving simultaneously the time derivative of the potential and the body acceleration, in adding the equation of motion in the linear system to be solved for the BVP.

\section{Numerical Validation}

In [1], we presented results for a submerged sphere in forced motion. Here, numerical results for the free response of a submerged sphere are shown.

\section{Description of the test case (figure 1)}

An elastic spring, with a stiffness coefficient of $k=$ $50000 \mathrm{~N} . \mathrm{m}^{-1}$, is applied to the center of gravity of a sphere of radius $a=2 m$. The fixed end of the spring is at a vertical position of $z_{s}=-2 a$ below the free-surface.

The sphere, with a zero buoyancy, is set free at an initial vertical distance of $d=0.5 a$ from the spring fixed end.

The closed domain, cylindrical of radius $R=10 \mathrm{~m}$ and depth $H=10 m$, is deprived of any absorbing beach to ensure the energy conservation. No incident wave is applied, which implies that the free-surface equations are linearized on the mean position $z=0$.

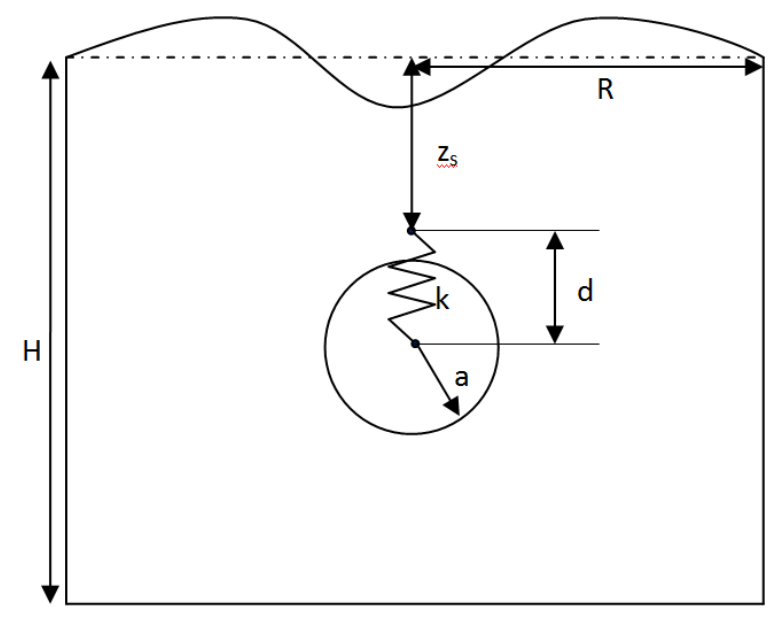

FIGURE 1. Test case schema

The fluid energy is given by

$$
\begin{aligned}
E_{\text {fluid }} & =E_{p}+E_{k}=\iiint_{D}\left(\frac{1}{2} \rho \mathbf{v}^{2}+\rho g z\right) d v \\
& =\frac{1}{2} \rho \iint_{S_{F}+S_{B}} \phi \frac{\partial \phi}{\partial n} d S+\frac{1}{2} \rho g \iint_{S_{F}} \eta^{2} d S
\end{aligned}
$$

and its variation in time is equal to the work of the force of the body $\mathbf{F}_{H}$ acting on the fluid

$$
\frac{d E_{\text {Fluid }}}{d t}=P_{\mathbf{F}_{H}}=-\mathbf{F}_{H} \cdot \dot{\mathbf{x}}_{B}
$$

\section{Results}

The vertical motion of the sphere is presented in figure 2 .

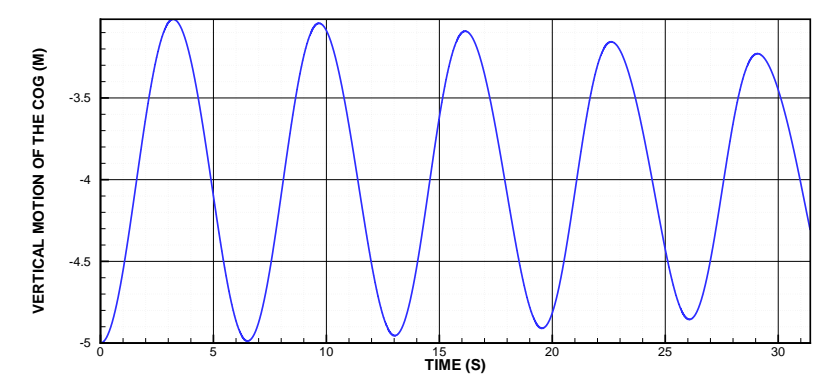

FIGURE 2. Vertical motion of the center of gravity of the sphere 
One can see the damped oscillations of the sphere, around its equilibrium position $z_{s}$, with a body motion period of $T=6.51 \mathrm{~s}$, and a logarithmic decrement of $\delta=6.4 \cdot 10^{-2}$.

The body motion creates a perturbation on the free surface, as seen in figure 3 for three different radial distances $r$ from the center of the free-surface : above the sphere at $r=0 m$, at $r=a$ and $r=2 a$.

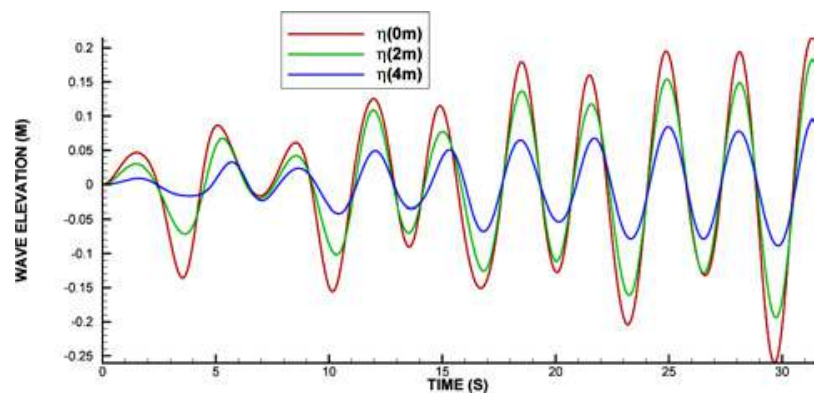

FIGURE 3. Wave elevation at different radius : $0 \mathrm{~m}, 2 \mathrm{~m}, 4 \mathrm{~m}$

The mean period of the wave elevation solutions is close to $T=3.4 s$, for the 3 positions. However non-linearities are easily seen on this plot, even if the propagation of the wave from one point to the next is difficult to analyze. Since the domain is quite small compared to the wavelength of the perturbation generated by the body, the reflections are present in the wave elevation solution after only a few seconds. However, this test case was set up to ensure the energy conservation was correct with free body motions.

One can see, in the figure 4, the energy conservation, compared to the energy in the closed fluid domain and the work of the hydrodynamic force.

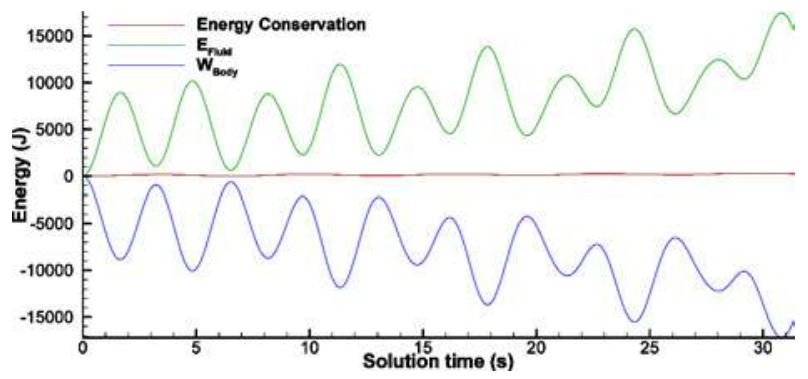

FIGURE 4. Energy Conservation of the fluid domain

The relative error is close to $\varepsilon=1.5 \%$ and constant during the simulation of the five periods of motion of the body. While the energy in the fluid domain is growing periodically, the error stays relatively equals to $1.5 \%$.

Several possibilities can explain the error obtained, even if said error can be considerated sufficiently small to validate the model. The supplementary term $q$ in the body condition for the $\mathrm{BVP}$ on $\phi_{t}$ requires second order spatial derivatives, difficult to compute precisely numerically. However a mesh convergence on the body was performed, and similar errors obtained. The second source of errors lies on the resolution of the free-surface mesh, which may not be small enough to capture short waves, high harmonics in the scattering solution. A mesh convergence was also performed which tends to validate this hypothesis. Solutions for rougher meshes on the free-surface were indeed of poorer precision. However a solution with a refined mesh on the free-surface could not be obtained, due to some instabilities generated by the local derivative calculations at the center of the free-surface mesh. A non structured mesh on the free-surface may thus give better results.

\section{PART II : DIFFRACTION AND RADIATION OF SURFACE PIERCING BODY}

\section{DISCRETISATION OF THE DOMAIN}

In order to be able to mesh complex geometry, an unstructured mesh is adopted (figure 5). The fluid domain geometry

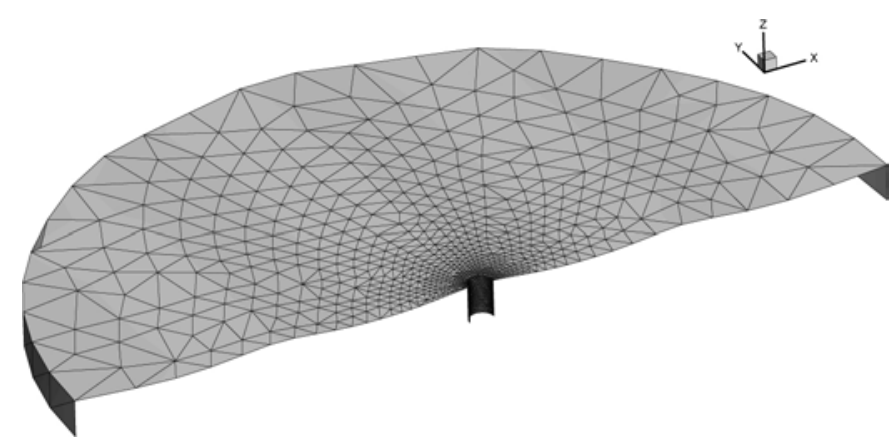

FIGURE 5. View of the unstructured mesh

changes with the elevation of the incident free surface and exact body position. It is thus necessary to adapt the mesh at each time step according to these deformations. In order to avoid remeshing the domain at each time step, (which would be time consuming and sources of numerical errors due to interpolation), mesh deformations algorithms have been integrated. In this way it is possible to keep the same mesh topology during the deformation and good quality mesh thanks to the arbitrary LagrangianEulerian method. However, for long duration of simulation, degenerated elements may appear through the deformation. In this 
case, a complete remeshing of the instantaneous wetted surface of the body and free surface is necessary. An advancing front method [11] has been integrated in such case. Shape and size of cell elements and the global mesh quality are controlled by quality metric functions. Combining mesh deformations algorithms and remeshing capabilities allows maintaining a good mesh quality over the whole duration of the simulation while minimising the computational time.

\section{Mesh deformation on the body}

Mesh on the wetted surface of the body is deformed with a spring analogy method. In this method, each edge of the mesh is modelled like a spring. Nodes are submitted to stress resulting on the compression and expansion of edges during deformation. The resulting stress applied on nodes can be defined from the effort of each edge connected to it and the displacement of its neighbours nodes.

$$
\mathbf{F}_{i}=\sum_{j=1}^{n_{v}} k_{i j}\left(\Delta \mathbf{x}_{i} \cdot \mathbf{n}_{i j}-\Delta \mathbf{x}_{j} \cdot \mathbf{n}_{i j}\right) \mathbf{n}_{i j}
$$

where $k_{i j}=\frac{1}{\left\|x_{i}-x_{j}\right\|^{2}}$ is the stiffness of the spring connecting nodes $i$ and $j, \mathbf{n}_{i j}$ is the direction of the spring and $n_{v}$ the number of neighbours nodes. Displacements $\Delta \mathbf{x}_{i}$ and $\Delta \mathbf{x}_{j}$ of the node $i$ and $j$ are described on the local base $\left(\mathbf{u}, \mathbf{v}, \mathbf{n}_{S M}\right)$.

$$
\Delta \mathbf{x}=d_{u} \cdot \mathbf{u}+d_{v} \cdot \mathbf{v}+d_{w} \cdot \mathbf{n}_{S M}
$$

with $\mathbf{n}_{S M}$ the unit normal vector to the surface, $\mathbf{u}$ and $\mathbf{v}$ two unit vectors tangential to the surface of the body and $\left(\mathbf{u}, \mathbf{v}, \mathbf{n}_{S M}\right)$ orthogonal and direct.

The zero flux boundary condition on the body surface implies that

$$
d_{w}=\left(\frac{D \mathbf{x}_{B}}{D t}+\omega \times\left(\mathbf{x}-\mathbf{x}_{B}\right)\right) \cdot \mathbf{n}_{S M} d t
$$

Besides, nodes on the edge between two surfaces should remain on this edge. Thus, nodes located at the intersection of the free surface and the body-interface should remain at this intersection. The local base is defined such as $\mathbf{n}_{S M}$ is normal to one surface, $\mathbf{u}$ is tangential to the edge and $\mathbf{v}$ is chosen in order that the local reference frame created with this three vectors is orthogonal and direct. These condition implies a condition on $d_{v}$.

$$
d_{v}= \begin{cases}\frac{\partial \phi_{0}}{\partial n_{S L}} \mathbf{n}_{S L} \cdot \mathbf{v} d t & \text { on the water line } \\ \left(\frac{D \mathbf{x}_{B}}{D t}+\omega \times\left(\mathbf{x}-\mathbf{x}_{B}\right)\right) \cdot \mathbf{v} d t & \text { in other case }\end{cases}
$$

with $\mathbf{n}_{S L}$ the normal vector on the free-surface.

The displacement of nodes located at the intersection of at least three surfaces is completly defined as it should remain at this intersection.

When the equilibrium is reached, the resulting force on nodes is equal to 0 . Thus, one can write a system of equations with size $2 n_{e}$ with $n_{e}$ being the number of nodes on the wetted surface of the body.

$$
\begin{aligned}
& \mathbf{F}_{i} \cdot \mathbf{u}=0 \\
& \mathbf{F}_{i} \cdot \mathbf{v}=0
\end{aligned}
$$

The resulting linear sparse matrix equation $A X=b$ is solved using LU factorisation algorithm.

\section{Deformation of the free surface mesh}

The previous method is not applicable for the free surface because the size of the linear system would become too large. Instead, an interpolation scheme for the deformation is adopted [4]. The deformation function $s$ is a function of the position of nodes and a sum of basis functions.

$$
\mathbf{s}(\mathbf{x})=\sum_{j=1}^{n_{b}} \alpha_{j} \Psi\left(\left\|\mathbf{x}-\mathbf{x}_{j}\right\|\right)+p(\mathbf{x})
$$

with $n_{b}$ the number of structural node on the boundary interface, $\Psi$ a radial basis function and $p$ a polynomial of degree 1. In the following sections we use the radial basis function $\Psi(x)=x^{2} \log (x)$. Coefficient $\alpha_{j}$ are determined by the interpolation conditions for nodes at the intersection of the body and the free surface and at the intersection of the free surface and the outer cylinder limiting the fluid domain.

$$
\mathbf{s}\left(\mathbf{x}_{b j}\right)=\mathbf{d}_{b j}
$$

An additional requirement is needed to estimate the coefficient of the polynomial $p$ :

$$
\sum_{j=1}^{n_{b}} \alpha_{j} q\left(\mathbf{x}_{j}\right)=0
$$

where $\mathbf{d}_{b j}$ is the displacement of the jth node and $q$ is a polynomial with a degree less or equal to the degree of polynomial $p$.

The size of the linear system to be solved is equal to the number of nodes at the intersections. It is much smaller than the total number of nodes on the free surface. 


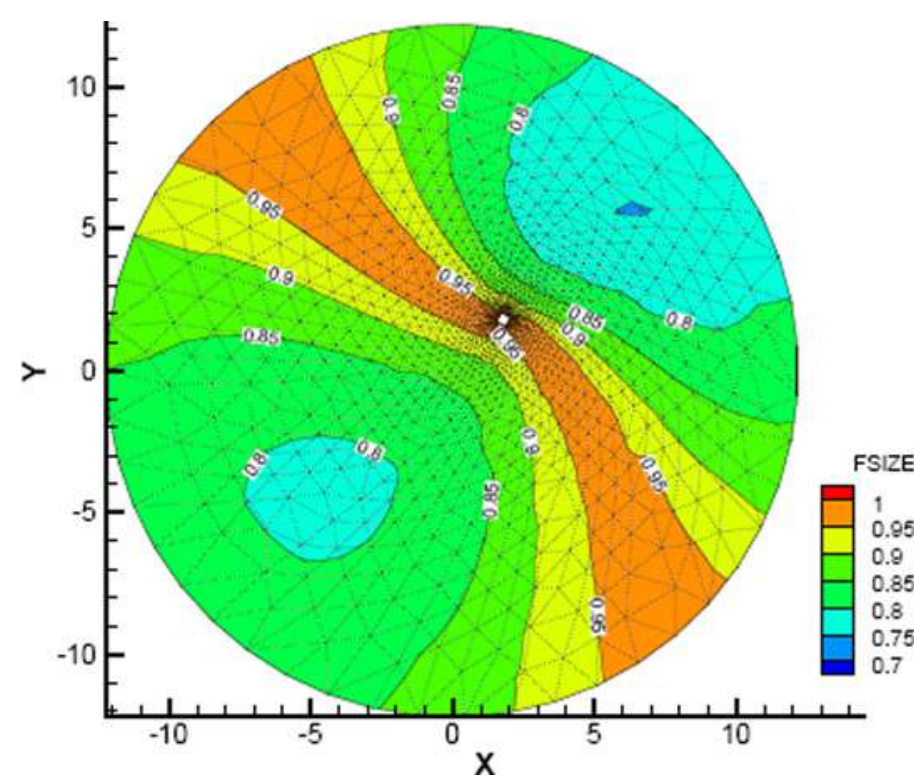

FIGURE 6. Size metric $f_{\text {size }}$ after deformation

The horizontal velocity of the nodes are obtained by numerical differentiation. An additional condition on the mesh velocity is needed to ensure that the node on the free surface stays at this interface.

$$
\mathbf{v}_{m e s h} \cdot \mathbf{n}=\frac{\partial \phi_{0}}{\partial n}
$$

on the free-surface.

The mesh quality can be evaluated through metric functions $f_{\text {size }}$ and $f_{\text {shape }}$ reflecting the size and shape of elements. A detailed review of possible metrics is presented in [12]. $f_{\text {size }}=1$ when an element keeps the same size after deformation whereas $f_{\text {size }}=0$ when an element becomes degenerated. $f_{\text {shape }}=1$ when the shape of the element is ideal (corresponding to an equilateral triangle for triangular meshes) whereas $f_{\text {shape }}=0$ when an element becomes degenerated. The conservation of these two metrics represents a good tool for monitoring the mesh quality during the deformation.

For sake of illustration, the mesh deformation for a free surface piercing cube of size $a$ in translation of $5 a$ in the $x$ and $y$ direction and in rotation of $\pi / 3$ in $z$ direction (the vertical direction) is considered.

Figure 6 shows the mesh and the contour field for the metric $f_{\text {size }}$ on the free surface after deformation. Minimal value of $f_{\text {size }}$ is about 0.7 which implies that no degenerated cell has been created. In the vicinity of the cube, $f_{\text {size }}$ is close to the maximum value 1 which means that sizes of those elements are not altered by the translation. Cells close to the cube are translated without deformation. In consequence, mesh refinement is well preserved by this mesh deformation algorithm. One can see that the maximum mesh deformation is absorbed close to the outer boundary limit of the domain along the axis of the displacement.

\section{SPATIAL SMOOTHING}

Instabilities known as saw-tooth instabilities generally appear close to the interface between the free surface and the body during time domain simulation of fluid-structure interaction. These instabilities are related to small wave length which are difficult to model accurately. Many methods have been developed. Most of them are based on the interpolation scheme from neighbouring nodes for a structural mesh $[13,14]$. Fewer methods have been developed for unstructured meshes. In this work, the method developed in [15] is used. It is a generalisation of a three point interpolation model. This low pass filter is applied for each node of the free surface mesh.

$$
\bar{f}_{0}=(1-c) f_{0}+\sum_{j=1}^{N} w_{j} f_{j}
$$

with $f_{0}$ being the value at the node before smoothing and $\bar{f}_{0}$ the value after smoothing. Weighting coefficients $w_{j}$ depend on the distance $l_{j}$ between the $j$ th neighbour node and the selected node and $c$ the strengh of the filter.

$$
w_{i}=c \frac{l_{i}^{2}}{\sum_{N} l_{j}^{2}}
$$

The low pass filter is applied every 5 steps in order to limit its effect on the numerical accuracy.

\section{NUMERICAL RESULTS}

\section{Diffraction by a vertical truncated cylinder}

Wave runup for a vertical truncated cylinder is first studied in this section. Runups are compared to numerical results of the fully nonlinear potential flow model Xwave developped by Ferrant [16]. The incident nonlinear regular wave is obtained from the stream function theory of Rienecker \& Fenton [17] and has a wave period of $1.8 s$. The domain is a circular cylinder of $2 \lambda_{0}$ radius (with $\lambda_{0}$ the wave lenght of the incident wave) and of finite depth $h=10 \mathrm{~m}$. An absorption zone is defined on the outer region of the domain and extended over one wavelength. Characteristics of the domain give the following non-dimentional parameters $k h=11.9, k R=0.372,2 a / \lambda_{0}=0.046$ (wave steepness 

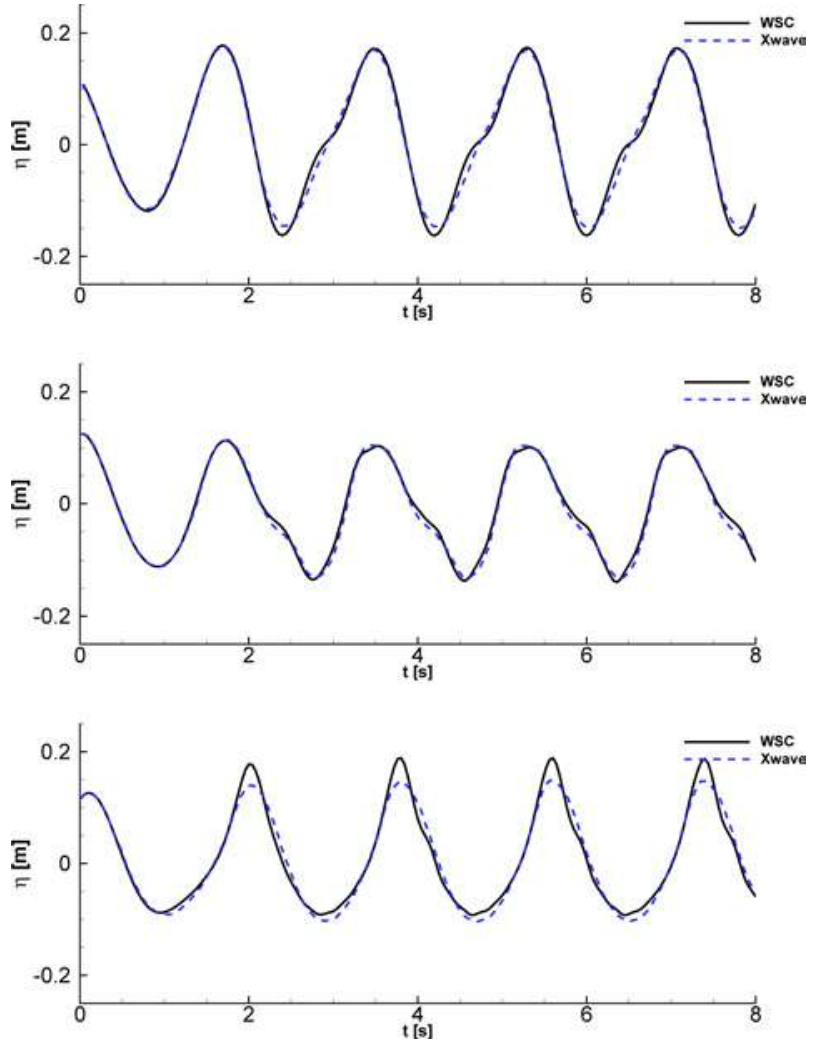

FIGURE 7. Time traces of the runup around the cylinder $(\theta=\pi$ on top, $\theta=\pi / 2$ on the midle, $\theta=0$ on bottom). Comparison of numerical result with the weak-scatterer approach (black) and the fully nonlinear model Xwave (blue).

of $4.6 \%$ ), with $R$ being the radius of the internal cylinder and $a$ being the wave amplitude. The cylinder in the center of the domain extends to $1 \mathrm{~m}$ below the still water level. The mesh contains 2241 elements with a finer resolution in the close vicinity of the body. The refinement is used because wave scattering is generated close to the body and is dissipated at the outer regions. The time step for the simulation is fixed. It is equal to $0.04 s$.

Time traces of the runups are shown in figure 7 for three differents positions around the cylinder corresponding to the bow side $(\theta=\pi)$, the side $(\theta=\pi / 2)$ and the lee side $(\theta=0)$. One can see that results from the weak-scatterer approach agree well with the nonlinear potential flow results for $\theta=\pi$ and $\theta=\pi / 2$. However, we can notice that the weak-scatterer approach underestimates the crest of the wave elevation on the bow side of the cylinder. On the lee side, one can see that the general shape of the runup is correctly predicted by the model but the weak-scatterer approximation overestimates the maximum runups in comparison with the nonlinear potential flow results. All nonlinearities are not taken into account by the weak-scatterer approximation

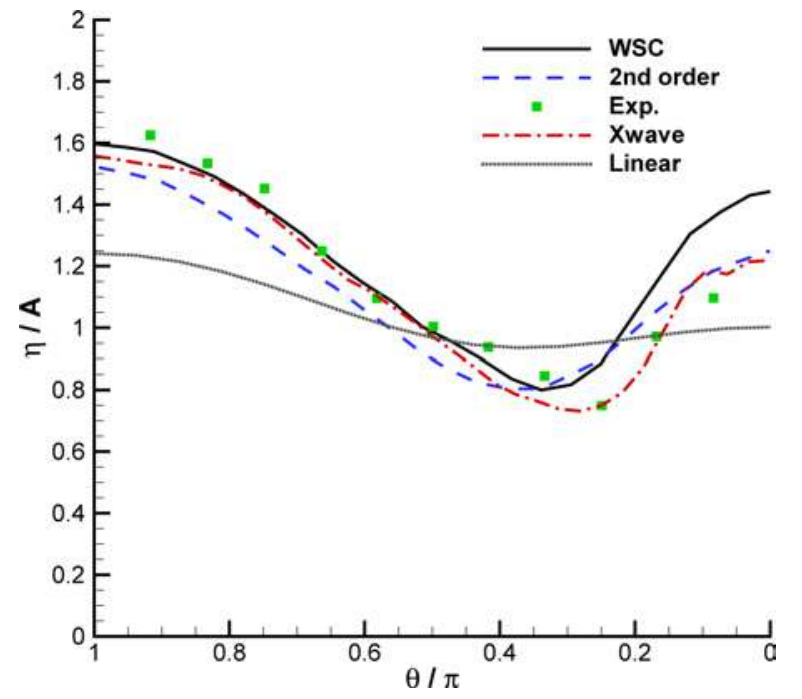

FIGURE 8. Maximum runup around the cylinder for a wave steepnexx of $3.2 \% . k R=0.374, k h=1.036$. Comparison of weak-scatterer results (WSC), second-order theory, experimental data, linear and nonlinear Xwave [20]

which explains those differences.

\section{Diffraction by a bottom-mounted vertical cylinder}

In this section, the diffraction by a bottom-mounted vertical cylinder is studied. This configuration has been widely studied. Thus, it is an interesting benchmark to validate the present model. Experimental data is available for different wave steepness in [8] and can be compared to numerical results. In the following simulation the incident nonlinear regular wave is defined by the stream function theory of Rienecker \& Fenton [17]. The configuration of the domain gives the following non dimentional parameters $k h=1.036, k R=0.374$ (with $R$ the radius of the internal cylinder). The domain extension is $2 \lambda_{0}$ radius length with $\lambda_{0}$ the wave length of the incident wave. Two differents wave amplitudes are simulated corresponding to a wave steepness of $3.2 \%$ and $4.6 \%$. Maximum runups around the cylinder are represented in figure 8 and figure 9 for a wave steepness of 3.2\% and $4.6 \%$ respectively. They are compared to experimental data (Kriebel [8]), results from fully nonlinear model (Xwave [16]). Results from the second order theory (Pelletier [18]) and from the linear theory of MacCamy and Fuchs [19] are also shown.

In this test case, non linear effects are known to be important. Consequently, the linear model underestimates the maximum runup on the bow size of the cylinder (relative error of about $36 \%$ for the wave steepness of 3.2\%). The weak-scatterer approach and the nonlinear model Xwave agree well on the wea- 


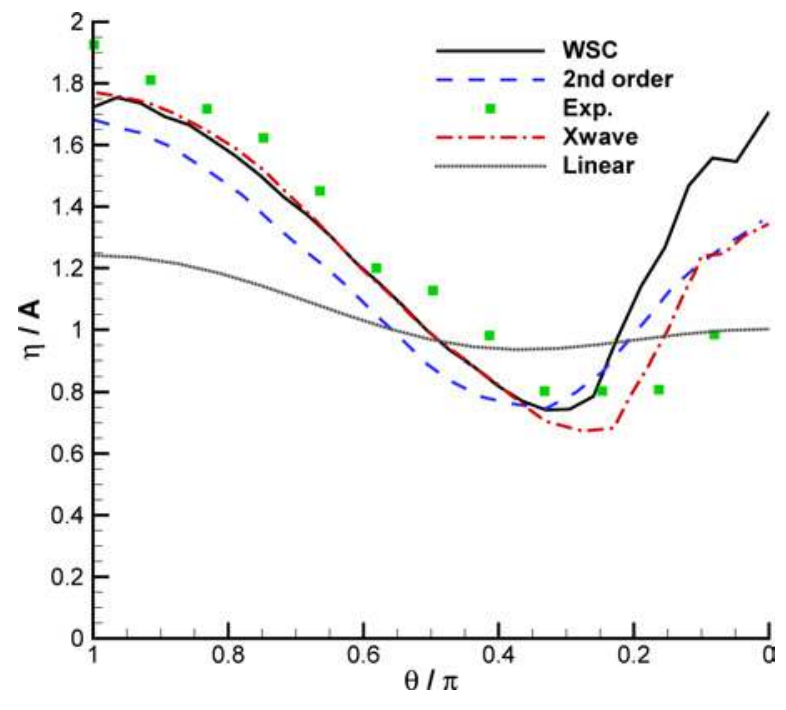

FIGURE 9. Maximum runup around the cylinder for a wave steepness of $4.6 \% . k R=0.374, k h=1.036$. Comparison of weak-scatterer results (WSC), second order theory, experimental data, linear and fully non linear model Xwave [20]

ther side of the cylinder for both steepnesses. For the first steepness, the relative error for the maximum runup on the bow side is about $6 \%$ for the weak-scatterer model. Besides the crest located near $\theta=\pi / 3$ is better predicted by the model. One can note that the weak-scatterer approach gives a better estimation of the maximum runup on the bow side than the second order model which underestimates the maximum runup. On the lee side, the weak-scatterer model overestimates the maximum runup compared to the fully nonlinear model and the second order model.

For a wave steepness of $4.6 \%$ the weak-scatterer approach underestimates the maximum runups on the bow side of the cylinder but remains close to the behaviour of the fully nonlinear model.

\section{Wave radiation by a truncated cylinder}

In the following section, radiated wave generated by a truncated cylinder are investigated and results are compared to a fully nonlinear model [21]. The incident wave is equal to zero. The radiated waves are generated by the motion of a cylinder in surge and pitch. The mesh topology is the same in both simulations and no remeshing is needed. For surge motion only the mesh of the free surface is deformed using the RBF interpolation method while for pitch motion, both mesh on the free surface and on the body are deformed (using RBF interpolation method for the free surface and spring analogy for the body).

The configuration of the domain leads to the following non-

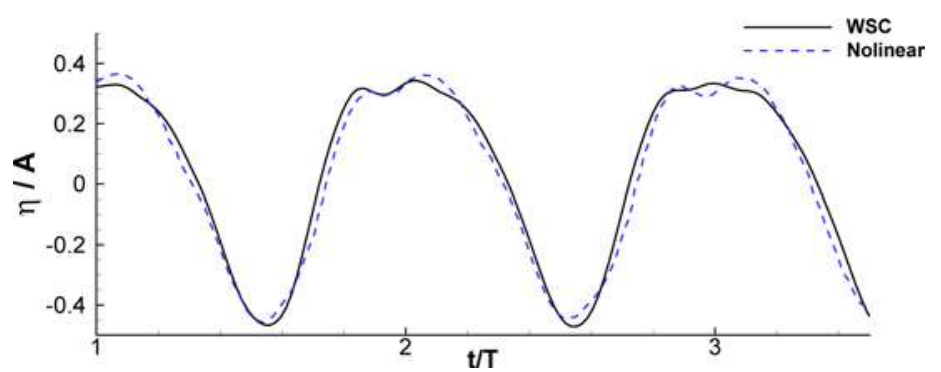

FIGURE 10. Wave runup evolution in $(-R, 0)$ with the cylinder in surge motion. $\omega \sqrt{d / g}=1.257, A=0.046 h$. Comparison of weakscatterer result (black) and nonlinear result (blue).

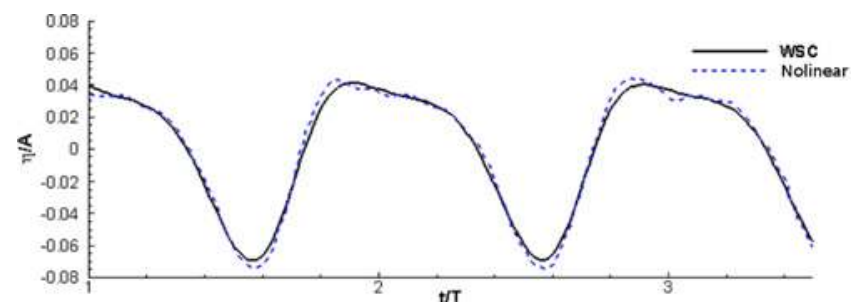

FIGURE 11. Wave runup evolution in $(-R, 0)$ with the cylinder in pitch motion. $\omega \sqrt{d / g}=1.257, A=\pi / 20$. Comparison of weakscatterer result (black) and nonlinear result (blue).

dimentional parameters $R / h=0.2$ and $d / h=0.5$ with $R$ the radius of the cylindrical body and $d$ its initial draft and $h$ the water depth. The motion of the cylinder is given by $\xi=-A \cos (\omega t)$ with $\omega$ the frequency and $A$ the amplitude. The frequency is defined by its non-dimensional expression $\omega \sqrt{d / g}=1.257$. The amplitude is equal to $A=0.046 h$ for surge motion and $A=\pi / 20$ for pitch motion.

In figure 10 and figure 11 runup at $(-R ; 0)$ is compared to numerical results obtained with the fully nonlinear model [21]. One can see that for surge motion and pitch motion both models agree well in their predictions. Some differences appear for the crests where the weak-scatterer may not include all the nonlinearities.

\section{CONCLUSION}

The hydrodynamic equations of the fluid-structure interaction model based on the weak-scatterer approximation have been presented. For free body motions, the instantanneous pressure computation on the wetted surface of the body has been introduced thanks to the resolution of a Green's Second identity equation on $\phi_{t}$. Cointe's [3] and Tanizawa's [2] formulations for the body condition have been revisited. The equation of motion is solved simultaneously with the BVP on the time derivative of the velocity potential using the implicit condition method. This me- 
thod has been validated by studying the energy conservation on a freely moving submerged sphere in a closed domain. The body motions and the wave perturbation on the free-surface have also been presented. Test cases have been simulated for the diffraction of a nonlinear regular wave of large steepness on a vertical cylinder piercing the free surface. These results have been compared to a fully nonlinear model Xwave and to experimental data. The capacity of the model to represent nonlinearity have been highlighted. We observe that the model gives results very close to a fully nonlinear model for the part in the front of the cylinder. All nonlinearities are not taken into account by the model and the model overestimates the maximum runup in the lee side of the cylinder.. The efficiency of this moving strategy has been tested on wave radiation simulation and compared to fully nonlinear results. The general behavior of the wave runup obtained with the weak-scatterer approximation is in good agreement with nonlinear results. Next, those methods will allow to simulate the body response of a free floating body with a more complex geometry with no vertical surface interface.

\section{Références}

[1] Letournel, L., Harris, J., Ferrant, P., Benoit, M., Babarit, A., Ducrozet, G., and Emmanuel, D., 2014. "Comparison of fully non-linear and weakly nonlinear potential flow solvers for the study of wave energy converters undergoing large amplitude motions". In 33rd International Conference on Ocean, Offshore and Artic Engineering.

[2] Tanizawa, K., 1995. "A Nonlinear Simulation Method of 3-D Body Motions in Waves (1st Report)". Journal of the Society of Naval Architects of Japan, 1995(178), pp. 179191.

[3] Cointe, R., 1989. "Quelques aspects de la simulation numérique d'un canal à houle". $\mathrm{PhD}$ thesis, Ponts et Chaussées.

[4] Boer, A., der Schoot, M. V., and Bijl, H., 2007. "Mesh deformation based on radial basis function interpolation". Computers and Structures, 85, pp. 784-795.

[5] Bhinder, M. A., Mingham, C. G., Causon, D. M., Rahmati, M. T., Aggidis, G. A., and Chaplin, R. V., 2009. "Absorbing wave energy converter ( WRASPA )". In 28th International Conference on Ocean, Offshore and Artic Engineering, pp. 1-7.

[6] The Seakeeping Committee, 2011. Final report and recommendations to the 26th ittc. Tech. rep.

[7] Pawlowski, J., 1991. "A nonlinear theory of ship motion in waves”. In 19th Symposium on naval Hydrodynamics.

[8] Kriebel, D., 1992. "Nonlinear wave interaction with a vertical circular cylinder. part ii : Wave run-up.". Ocean Engineering, 19(1), pp. 75-99.
[9] Van Dalen, and George, E., 1993. "Numerical and Theoretical Studies of Water Waves and Floating Bodies". PhD thesis.

[10] Berkvens, 1998. "Floating bodies interacting with water waves : Development of a time-domain panel method". $\mathrm{PhD}$ thesis, Universiteit Twente (The Netherlands.

[11] Frey, J., and George, P., 2000. ”Maillages : applications aux lements finis". Hermes.

[12] Stimpson, C., Ernst, C., Knupp, P., Pbay, P., and Thompson, D., 2007. The verdict geometric quality library. Tech. rep.

[13] Longuet-Higgins, M., and Cokelet, E., 1976. "The deformation of steep surface waves on water, i. a numerical method of computation". In Proceedings of the Royal Society of London, Vol. 350, pp. 1-26.

[14] Buchmann, B., 1999. "Time-domain modelling of run-up on offshore structures in waves and current". PhD thesis, Technical University of Denmark.

[15] Shao, Y., and Faltinsen, M., 2013. "Fully-nonlinear wavecurrent-body interaction analysis by a harmonic polynomial call (hpc) method". In 32nd International Conference on Ocean, Offshore and Artic Engineering.

[16] Ferrant, P., 1998. "Fully non-linear interactions of longcrested wave packets with a three-dimensional body.". In 22nd ONR Symposium on Naval Hydrodynamics, pp. 403415.

[17] Rienecker, M., and Fenton, J., 1981. "A fourier approximation method for steady water waves". Journal of Fluid Mech., 104, pp. 119-137.

[18] Pelletier, K., 2002. "Simulation au second ordre des interactions houle-structure en rgime instationnaire". $\mathrm{PhD}$ thesis, LHEEA, Ecole Centrale de Nantes.

[19] MacCamy, R., and Fuchs, R., 1954. "Waves forces on piles : A diffraction theory.". US Army Coastal Engineering Research Center, Tech Mem., 69.

[20] Ferrant, P., 1999. "Seakeeping calculations in nonlinear waves.”. In Proceedings of the 7th International Conference on Numerical Ship Hydrodynamics.

[21] Zhou, B., Ning, D., Teng, B., and Bai, W., 2013. "Numerical investigation of wave radiation by a vertical cylinder using a fully nonlinear hobem". Ocean Engineering, 70, pp. 1-13. 